\title{
Two-Dimensional Gallium Sulfide Nanoflakes for UV-Selective Photoelectrochemical-type Photodetectors
}

\author{
Marilena I. Zappia, Gabriele Bianca, Sebastiano Bellani,* Nicola Curreli, Zdeněk Sofer, Michele Serri, \\ Leyla Najafi, Marco Piccinni, Reinier Oropesa-Nuñez, Petr Marvan, Vittorio Pellegrini, Ilka Kriegel, \\ Mirko Prato, Anna Cupolillo, and Francesco Bonaccorso*
}

Cite This: J. Phys. Chem. C 2021, 125, 11857-11866

Read Online

ACCESS | 네 Metrics \& More | 回 Article Recommendations | sl Supporting Information

ABSTRACT: Two-dimensional (2D) transition-metal monochalcogenides have been recently predicted to be potential photo(electro)catalysts for water splitting and photoelectrochemical (PEC) reactions. Differently from the most established InSe, $\mathrm{GaSe}, \mathrm{GeSe}$, and many other monochalcogenides, bulk GaS has a large band gap of $\sim 2.5$ $\mathrm{eV}$, which increases up to more than $3.0 \mathrm{eV}$ with decreasing its thickness due to quantum confinement effects. Therefore, $2 \mathrm{D} \mathrm{GaS}$ fills the void between $2 \mathrm{D}$ small-band-gap semiconductors and insulators, resulting of interest for the realization of van der Waals type-I heterojunctions in photocatalysis, as well as the development of UV light-emitting diodes, quantum wells, and other optoelectronic devices. Based on theoretical calculations

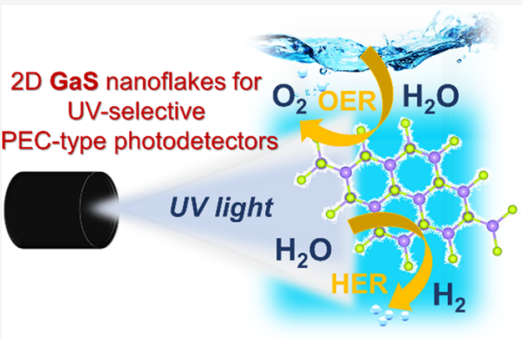
of the electronic structure of $\mathrm{GaS}$ as a function of layer number reported in the literature, we experimentally demonstrate, for the first time, the PEC properties of liquid-phase exfoliated GaS nanoflakes. Our results indicate that solution-processed 2D GaS-based PEC-type photodetectors outperform the corresponding solid-state photodetectors. In fact, the $2 \mathrm{D}$ morphology of the GaS flakes intrinsically minimizes the distance between the photogenerated charges and the surface area at which the redox reactions occur, limiting electron-hole recombination losses. The latter are instead deleterious for standard solidstate configurations. Consequently, PEC-type $2 \mathrm{D} \mathrm{GaS}$ photodetectors display a relevant UV-selective photoresponse. In particular, they attain responsivities of $1.8 \mathrm{~mA} \mathrm{~W}^{-1}$ in $1 \mathrm{M} \mathrm{H}_{2} \mathrm{SO}_{4}$ [at $0.8 \mathrm{~V}$ vs reversible hydrogen electrode (RHE)], $4.6 \mathrm{~mA} \mathrm{~W}^{-1}$ in $1 \mathrm{M}^{-1}$ $\mathrm{Na}_{2} \mathrm{SO}_{4}$ (at $0.9 \mathrm{~V}$ vs RHE), and $6.8 \mathrm{~mA} \mathrm{~W}^{-1}$ in $1 \mathrm{M} \mathrm{KOH}$ (at 1.1. V vs RHE) under $275 \mathrm{~nm}$ illumination wavelength with an intensity of $1.3 \mathrm{~mW} \mathrm{~cm}^{-2}$. Beyond the photodetector application, 2D GaS-based PEC-type devices may find application in tandem solar PEC cells in combination with other visible-sensitive low-band-gap materials, including transition-metal monochalcogenides recently established for PEC solar energy conversion applications.

\section{INTRODUCTION}

Gallium sulfide $(\mathrm{GaS})$ is a binary IIIA-VIA group compound, which has gained increasing attention among the plethora of layered semiconductors due to its distinctive optoelectronic and anisotropic structural properties. ${ }^{1-3}$ Depending on the stacking of layers, four $\mathrm{GaS}$ polytypes $(\beta, \varepsilon, \gamma$, and $\delta)$ are distinguished, ${ }^{4}$ although the hexagonal ( $2 \mathrm{H}$ phase) $\beta$ polytype $^{5,6}$ is the most energetically favorable crystal arrangement. ${ }^{4}$ A single layer of $\beta-\mathrm{GaS}$ is composed of $\mathrm{S}-\mathrm{Ga}-\mathrm{Ga}-\mathrm{S}$ repeating units, with different layers kept together along the $c$ axis by weak van der Waals forces. ${ }^{7,8}$ Differently from other investigated transition-metal monochalcogenides (e.g., $\mathrm{GaSe},{ }^{9,10} \mathrm{InSe}^{11,12} \mathrm{GeSe}^{13}$ and $\left.\mathrm{SnSe}^{14}\right)$, the bulk form of $\mathrm{GaS}$ has a large optical band gap $\left(E_{\mathrm{g}}\right)$ (at $300 \mathrm{~K}$ : indirect $E_{\mathrm{g}} \sim$ $2.5 \mathrm{eV}^{15-18}$ direct $\left.E_{\mathrm{g}} \sim 3.0 \mathrm{eV}^{\mathrm{P} 7-19}\right)$. The $E_{\mathrm{g}}$ drastically increases above $3 \mathrm{eV}$ with decreasing thickness down to the monolayer state due to quantum confinement effects. ${ }^{20,21}$ Therefore, two-dimensional (2D) GaS fills the void between $2 \mathrm{D}$ small- $E_{\mathrm{g}}$ semiconductors and insulators, which is of interest for the realization of ultraviolet (UV)-selective photodetectors, $^{22-24}$ color-tuneable blue/UV light-emitting diodes
(LEDs), ${ }^{20}$ and van der Waals type-I heterojunctions in photocatalysis. $^{21,25-27}$ Meanwhile, 2D GaS emerged as a potential material for applications such as electrochemical water splitting, ${ }^{28}$ hydrogen storage, ${ }^{29}$ energy storage (e.g., Liion batteries), ${ }^{30,31}$ gas sensing, ${ }^{32,33}$ DNA sequencing, ${ }^{34}$ and nonlinear optics. ${ }^{35,36}$ Contrary to several 2D materials, which are reactive to air (e.g., elemental analogue of graphene, such as silicene, germanene, and stanene $e^{37,38}$ as well as transitionmetal tellurides ${ }^{39,40}$ ) or undergo photoinduced oxidation (e.g., phosphorene $e^{41-43}$ and metal monochalcogenides such as $\mathrm{GaSe}^{44-47}$ and $\mathrm{GeSe}^{48}$ ), nearly ideally stoichiometric 2D $\mathrm{GaS}$ is oxidation-resistant under both laser/strong UV illumination $^{22}$ and mechanical stress, ${ }^{49,50}$ showing a high

Received: April 21, 2021

Revised: April 28, 2021

Published: May 26, 2021 
(a)

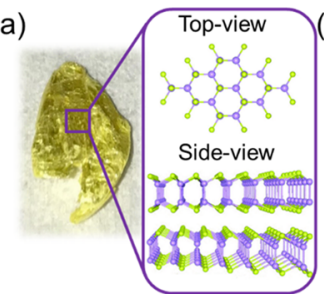

(d)

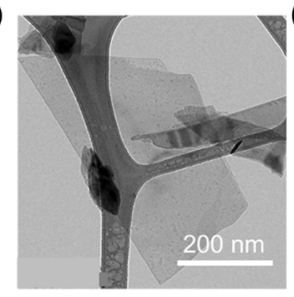

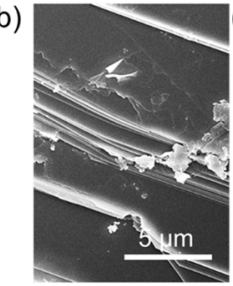
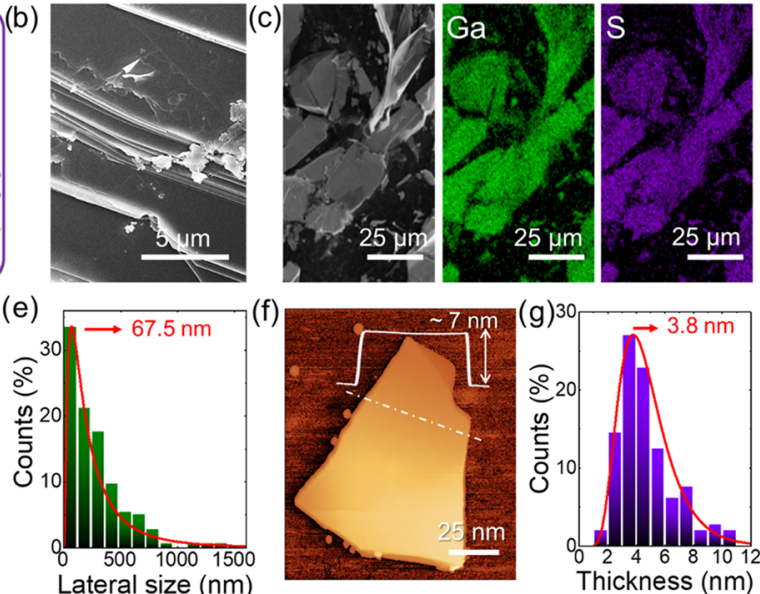

Figure 1. (a) Photograph of a $\beta-\mathrm{GaS}$ crystal synthesized through the direct reaction of $\mathrm{Ga}$ and $\mathrm{S}$ elements. The $2 \mathrm{H}$ structure $\left(P 6_{3} / m m c\right)$ of the crystal polytype is also shown. (b) SEM image of a fragment of the GaS crystal, showing its layered structure. (c) SEM image of fragments of GaS crystals and the corresponding EDS maps for $\mathrm{Ga}(\mathrm{K} \alpha=9.3 \mathrm{keV}$, green) and $\mathrm{S}(\mathrm{K} \alpha=2.3 \mathrm{keV}$, violet). (d) Bright-field transmission electron microscopy (BF-TEM) image of representative GaS flakes produced by the LPE of fragmentized GaS crystals. (e) BF-TEM statistical analysis of the lateral dimension of the GaS flakes. (f) Atomic force microscopy (AFM) image of a representative GaS flake. The height profile of a flake section is also shown. (g) AFM statistical analysis of the thickness of the GaS flakes.

activation energy $(\sim 3.1 \mathrm{eV})$ for the dissociation and chemisorption of $\mathrm{O}_{2}$ molecules. ${ }^{51}$

Despite the appealing features of $2 \mathrm{D} \mathrm{GaS}$, its optoelectronic properties have been mainly investigated in solid-state photodetectors based on isolated flakes, ${ }^{22,23,32}$ which were produced through mechanical cleavage $e^{23,32}$ or chemical vapor deposition. ${ }^{22}$ However, these techniques suffer from intrinsic scalability limits for their use in massive applications. ${ }^{52,53}$ Liquid-phase exfoliation (LPE) methods can provide scalable production of $2 \mathrm{D}$ materials in the form of a liquid dispersion, ${ }^{53-55}$ enabling their processing into thin films through low-cost and scalable deposition techniques, ${ }^{12,56-58}$ including roll-to-roll printing. ${ }^{59}$ Although the LPE represents a viable approach to exploit $2 \mathrm{D} \mathrm{GaS},{ }^{28,33}$ the printing of these materials produces percolating networks of flakes, which can inevitably lead to poor performances of optoelectronic devices compared to those measured for isolated flakes. ${ }^{12,60}$ This effect is ascribed to the high contact resistance between flakes compared to the intrinsic resistance of the flakes themselves. ${ }^{12,60-63}$ Therefore, it is pivotal to provide a paradigm shift in the design of printed optoelectronic devices to fully exploit the unique properties of solution-processed $\mathrm{GaS}$ flake films. In this context, ground-breaking experimental works demonstrated that group-IIIA monochalcogenides, including $\mathrm{GaS}$, display electrochemical activities toward the hydrogen evolution reaction (HER), even though at high overpotentials (typically $>0.4 \mathrm{~V}) .{ }^{11,28}$ Meanwhile, their $2 \mathrm{D}$ forms have emerged as potential photo(electro)catalysts for both water splitting reactions, i.e., HER and oxygen evolution reaction (OER), ${ }^{9,21,27}$ allowing new types of photoelectrochemical (PEC)-type photodetectors to be designed. ${ }^{9}$ Importantly, the number of their layers controls the energy of the conduction band minimum and valence band maximum $\left(E_{\mathrm{CBM}}\right.$ and $E_{\mathrm{VBM}}$, respectively). Consequently, group-IIIA monochalcogenides can be engineered to fulfill the fundamental requirements for the water splitting photo(electro)catalysts, ${ }^{13,21,27}$ i.e., (1) $E_{\text {СвM }}$ $>$ reduction energy level of $\mathrm{H}^{+} / \mathrm{H}_{2}\left(E\left(\mathrm{H}^{+} / \mathrm{H}_{2}\right)\right)$ and (2) $E_{\mathrm{VBM}}$ $<$ reduction energy level of $\mathrm{O}_{2} / \mathrm{H}_{2} \mathrm{O}\left(E\left(\mathrm{O}_{2} / \mathrm{H}_{2} \mathrm{O}\right)\right)$. ${ }^{64-66}$ Despite the existence of few experimental studies on the PEC properties of the most established monochalcogenides, such as
InSe, ${ }^{67} \mathrm{GaSe},{ }^{9}$ and $\mathrm{GeSe},{ }^{13}$ no PEC characterizations have been reported for $\mathrm{GaS}$, which is, therefore, a subject matter of interest for the realization of UV-harvesting components in photocatalytic tandem structures (e.g., van der Waals type-I heterojunctions). In this context, the double peak feature around the $\Gamma$-point in the so-called "Mexican-hat-like" ringshaped valence band dispersion of single-/few ( $\leq 5)$-layer GaS flakes effectively enhances the photoabsorption cross section. In fact, the electrons available for the optical transitions are twice those available in the case of a single-peak valence band minimum. ${ }^{23,27,68}$ Thanks to the 2D nature of GaS flakes, electrons and holes are directly photogenerated at the interface with the electrolyte, where redox reactions take place before the charges recombine. ${ }^{69-72}$ This last feature avoids the need for high-mobility active materials, which are instead mandatory for high-responsivity solid-state photodetectors. ${ }^{70,73,74}$

By rationalizing the above observations, we report for the first time the use of $2 \mathrm{D} \mathrm{GaS}$ flakes for the realization of quasivisible-blind UV-selective PEC-type photodetectors operating in aqueous media.

\section{METHODS}

The methods concerning materials, synthesis, and exfoliation of $\mathrm{GaS}$ crystals, materials characterization, fabrication of the photoelectrochemical (PEC)-type and solid-state photodetectors, and characterization of the photodetectors are reported in the Supporting Information (SI).

\section{RESULTS AND DISCUSSION}

Materials Characterization. GaS single-/few-layer flakes were produced through ultrasonication-assisted LPE of bulk $\beta$ polytype in eco-friendly anhydrous 2-propanol (IPA) (see SI, Methods section). ${ }^{75}$ The use of IPA as the exfoliating solvent circumvents the processability drawbacks related to the use of high-boiling-point and toxic solvents often used for the exfoliation of layered materials, ${ }^{76-78}$ e.g., $N$-methyl-2-pyrrolidone (NMP) for graphene ${ }^{79,80}$ and several metal chalcogenides. ${ }^{81}$ Figure la shows a photograph of a representative GaS crystal, together with the top- and side-views of its hexagonal 
double-layered structure with the $\mathrm{Ga}-\mathrm{Ga}$ and $\mathrm{Ga}-\mathrm{S}$ distances of 2.48 and $2.37 \AA$, respectively, ${ }^{82,83}$ and an interlayer distance approximating the monolayer thickness of $\sim 0.75 \mathrm{~nm} .{ }^{83}$ Figure $1 \mathrm{~b}$ shows the scanning electron microscopy (SEM) image of a fragment of the GaS crystal, evidencing its layered structure. The SEM-coupled energy-dispersive X-ray spectroscopy (EDS) analysis (Figure 1c and Table S1) indicates a nearly ideal stoichiometry of the GaS crystal (Ga-to-S atomic ratio $1.05)$.

Figure 1d shows the bright-field transmission electron microscopy (BF-TEM) image of a representative LPEproduced $\mathrm{GaS}$ flake, which displays a nearly rectangular shape with regular edges. The lateral sizes of the flakes follow a log-normal distribution peaked at $67.5 \mathrm{~nm}$ (Figure 1e). Figure if shows the atomic force microscopy (AFM) image of a representative exfoliated flake together with its height profile, corresponding to a thickness of $\sim 7 \mathrm{~nm}$. Figure S1 shows another AFM image displaying few-layer GaS flakes with thickness ranging between 1.9 and $4.3 \mathrm{~nm}$. The statistical analysis of the thickness data reports values ranging from 1.5 to $12 \mathrm{~nm}$, whose distribution is fitted by a log-normal curve peaked at $3.8 \mathrm{~nm}$ (Figure $1 \mathrm{~g}$ ). The experimental AFM thickness of monolayer GaS being between 0.85 and 1 $\mathrm{nm},{ }^{20,22,84}$ close to the theoretical value of $0.75 \mathrm{~nm},{ }^{83,84}$ our $\mathrm{GaS}$ flakes mainly consist of few ( $\leq 5)$-layer flakes.

Figure 2a shows the optical extinction spectrum of the LPEproduced $\mathrm{GaS}$ flake dispersion. The plot monotonically
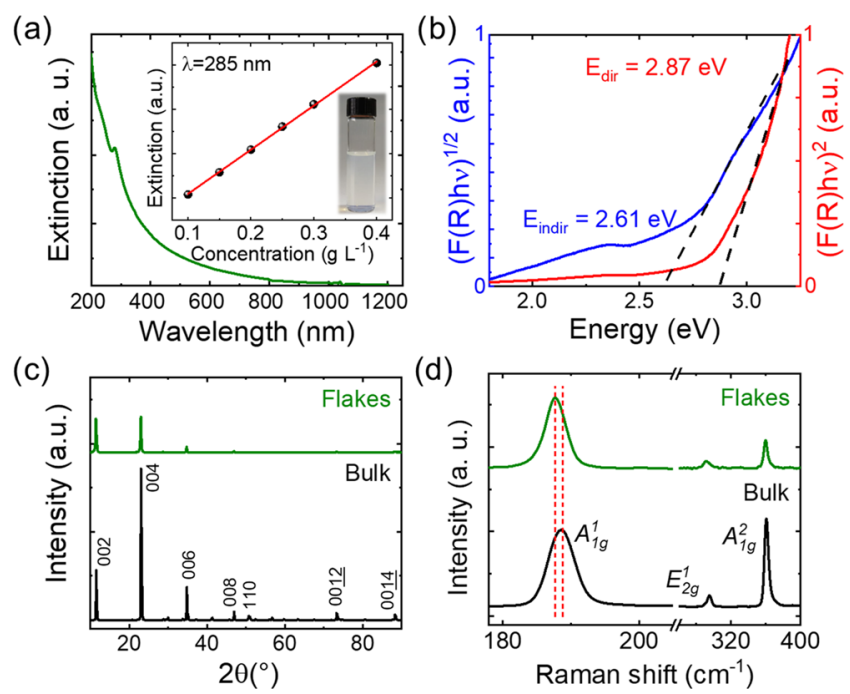

Figure 2. (a) Optical extinction spectrum of the LPE-produced GaS flake dispersion. The inset shows the $\operatorname{Ext}(285 \mathrm{~nm})$ vs $c$, together with a photograph of a GaS flake dispersion. (b) $(F(R) h \nu)^{n}$ vs $h \nu$ (Tauc plots) measured for the GaS flakes for direct $(n=2$, red trace) and indirect $(n=0.5$, blue trace) interband transitions. (c) XRD patterns and (d) Raman spectra $\left(\lambda_{\text {exc }}=514 \mathrm{~nm}\right)$ of bulk (black curve) and exfoliated (green curve) GaS crystals. Panels (c) and (d) report the diffraction peaks and Raman modes attributed to the $2 \mathrm{H}$ structure of $\beta$-GaS, respectively.

increases with decreasing wavelength until a narrow peak around $\sim 280 \mathrm{~nm}$ is reached. The tail of the spectrum in the visible and near-IR spectral region has been previously ascribed to the scattering contribution of dispersed flakes with nanoscale dimensions. ${ }^{28}$ The concentration of the as-produced $\mathrm{GaS}$ flake dispersion was first measured by weighing the solid material content in a known volume of dispersion, giving a value of $\sim 0.2 \mathrm{~g} \mathrm{~L}^{-1}$. The extinction coefficient of the GaS flakes was estimated using the Lambert-Beer law, $\operatorname{Ext}(\lambda)=$ $\varepsilon(\lambda) c l$, in which $\lambda$ is a given optical wavelength, $\operatorname{Ext}(\lambda)$ is the optical extinction at $\lambda, \varepsilon(\lambda)$ is the extinction coefficient at $\lambda, c$ is the material concentration, and $l$ is the optical path length. ${ }^{85}$ By measuring the optical extinction spectra of the as-produced $\mathrm{GaS}$ flake dispersions with controlled concentrations, $\varepsilon(285$ $\mathrm{nm}$ ) is found to be $\sim 199 \mathrm{~L} \mathrm{~g}^{-1} \mathrm{~m}^{-1}$. To guarantee the reproducibility of the material deposition processes, the concentration among different batches of $\mathrm{GaS}$ flake dispersion has been finely controlled by monitoring their optical extinction spectrum, i.e., $c=\operatorname{Ext}(\lambda) /(\varepsilon(\lambda) l)$. The $E_{\mathrm{g}}$ of the $\mathrm{GaS}$ flakes was determined by diffuse reflectance spectroscopy (DRS) of a film of GaS flakes, deposited through spray coating, using the Kubelka-Munk theory of diffuse reflectance $(R){ }^{86,87}$ In particular, the $E_{\mathrm{g}}$ can be estimated by fitting the linear part of $(F(R) h \nu)^{n}$ vs $h \nu$ (Tauc plot) with $(F(R) h \nu)^{n}=Y\left(h \nu-E_{\mathrm{g}}\right)$ (Tauc relation), in which $F(R)$ is the Kubelka-Munk function, defined as $F(R)=(1-R)^{2} / 2 R, h$ is Planck's constant, $\nu$ is the photon's frequency, and $Y$ is a proportionality constant. ${ }^{86,87}$ The value of $n$ specifies the type of the electronic transitions, distinguishing between direct $(n=2)$ and indirect interband transitions $(n=0.5){ }^{88-90}$ Figure $2 \mathrm{~b}$ shows the Tauc plots of the GaS flake film for both $n=2$ and 0.5 . The estimated direct $E_{\mathrm{g}}$ is $\sim 2.9 \mathrm{eV}$, while the indirect $E_{\mathrm{g}}$ is $\sim 2.6 \mathrm{eV}$. These $E_{\mathrm{g}}$ values agree with those reported in the literature for few-layer $\mathrm{GaS}$ flakes. $^{20}$ It is noteworthy that the sprayed GaS-based films consist of flakes with polydisperse morphological features. Therefore, the energy onset referring to $E_{\mathrm{g}}$ of the thickest nanoflakes may experimentally hide those of the thinnest ones, since the latter show higher $E_{\mathrm{g}}$ (theoretical values $>3$ $\left.\mathrm{eV}^{21,27}\right){ }^{20}$ Figure $2 \mathrm{c}$ reports the $\mathrm{X}$-ray diffraction (XRD) pattern of the $\mathrm{GaS}$ flakes in comparison with the one measured for GaS crystal powder. The XRD peaks of the exfoliated sample resemble those of the bulk crystals, which are indexed to the $2 \mathrm{H}$ structure of $\beta$-GaS (ICSD-173940) with lattice parameters $a=b=3.627 \AA$ and $c=17.425 \AA .^{91}$ The absence of characteristic peaks attributed to crystalline impurities, such as $\mathrm{Ga}_{2} \mathrm{O}_{3}$, indicates that both the synthesis and the subsequent LPE of GaS crystals generate products with marginal defects. The structural properties of the bulk and exfoliated GaS crystals were further evaluated by Raman spectroscopy measurements. The group theory predicts six nondegenerate Raman active optical modes for the $P 6_{3} / m m c\left(D_{6 h}\right)$ space group of bulk $\beta$-GaS, i.e., $2 \mathrm{~A}_{1 \mathrm{~g}}+2 \mathrm{E}_{1 \mathrm{~g}}+2 \mathrm{E}_{2 \mathrm{~g}}{ }^{92-94}$ The most intense and investigated ones are $A_{1 g}^{1}, A_{1 g}^{2}$, and $E_{2 g}^{1}$ (the latter often includes the contribution of the nearby $\left.E_{19}^{2}\right) .{ }^{92,93}$ These modes are also observed in the exfoliated $\mathrm{GaS},{ }^{94,95}$ and their intensities decrease with the reduction of the number of layers. $^{22,94}$ In particular, for the noncentrosymmetric monolayer GaS (space group: $D_{3 h}$ ), $\mathrm{E}_{2 \mathrm{~g}}^{1}$ is typically indistinguishable from the Raman signal of the Si substrate. ${ }^{22,94}$ Recent studies demonstrated that the peak position of $\mathrm{A}_{1 \mathrm{~g}}^{1}$ is a trustworthy indicator for simple and fast determination of the thickness of the GaS flakes. ${ }^{2,94}$ In fact, the $\mathrm{A}_{1 \mathrm{~g}}^{1}$ peak is softened (redshifted) following a decrease of the number of layers due to the reduced impact of the interlayer interaction on phonon restoring forces. ${ }^{22,94}$ This phenomenon has also been detected in $\mathrm{GaSe}^{9}$ and other transition-metal dichalcogenides (e.g., $\left.\mathrm{MoS}_{2}\right){ }^{76,96}$ Figure $2 \mathrm{~d}$ shows the Raman spectra of both bulk and exfoliated $\mathrm{GaS}$ crystals measured with an excitation wavelength $\left(\lambda_{\text {exc }}\right)$ of $514 \mathrm{~nm}$. In agreement with the above consideration, the $A_{1 g}^{1}$ peak position for the GaS flakes is 
slightly red-shifted compared to the bulk case, indicating the successful exfoliation of the crystals through the LPE method. The quantitative statistical analysis of the $A_{1 g}^{1}$ peak position (calculated on 40 different spectra) is reported in Figure S2. In addition, Raman spectra of the GaS flakes do not exhibit characteristic peaks attributed to other crystalline species beyond $\mathrm{GaS}$ (e.g., $\mathrm{Ga}_{2} \mathrm{O}_{3}$, showing a pronounced Raman mode peak at $\left.\sim 200 \mathrm{~cm}^{-1}\right) .9^{97}$ Therefore, these results further support that the LPE process of GaS crystals carried out in IPA does not cause relevant oxidation effects, in agreement with the XRD measurements and the X-ray photoelectron spectroscopy (XPS) analysis (Figures S3 and S4). High-resolution TEM (HRTEM) analysis was performed to further examine the crystal structure of the LPE-produced GaS flakes. Figure 3a (a)

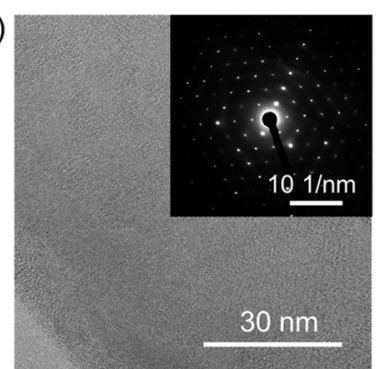

(c)

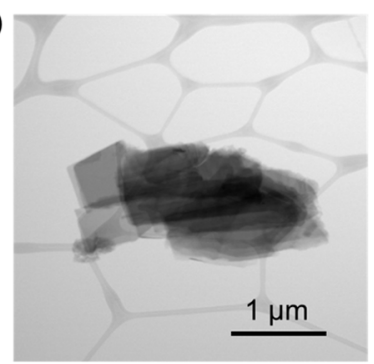

(b)

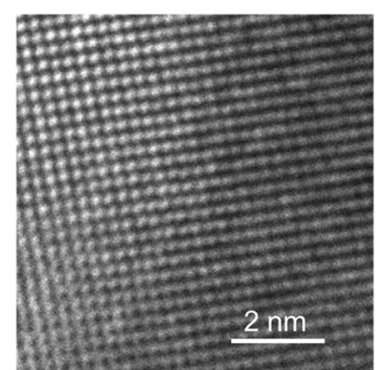

(d)

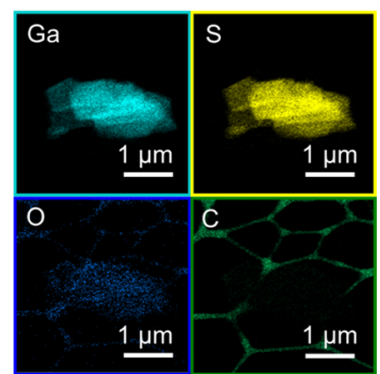

Figure 3. (a) BF-TEM image of a portion of a representative LPEproduced GaS flake near its edge. The inset shows the corresponding SAED pattern, which matches that of the $2 \mathrm{H}$ structure of $\beta$-GaS. (b) HRTEM image of a portion of the GaS flakes. (c) Scanning transmission electron microscopy (STEM) image of a partially suspended GaS flake. (d) Corresponding quantitative STEM-EDS

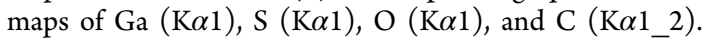

shows a BF-TEM image of a portion of a representative GaS flake near its edge. The corresponding selected area electron diffraction (SAED) pattern (inset of Figure 3a) matches that of the $2 \mathrm{H}$ structure of $\beta$-GaS (ICSD-173940), in agreement with the XRD analysis.

Figure $3 \mathrm{~b}$ shows the HRTEM image of a portion of the GaS flake, confirming the lattice spacings of the $\beta$-GaS. Scanning transmission electron microscopy coupled with EDS (STEMEDS) analyses were carried out to evaluate the composition of the GaS flakes. Figure 3c shows the STEM image of partially suspended $\mathrm{GaS}$ flakes. Figure $3 \mathrm{~d}$ shows the corresponding STEM-EDS maps of $\mathrm{Ga}, \mathrm{S}, \mathrm{O}$, and $\mathrm{C}$. The quantitative elemental analysis results in a $\mathrm{S}$-to-Ga atomic ratio of $\sim 1.1$ and a low atomic content of $\mathrm{O}$ (O-to-Ga atomic ratio $\sim 0.08$ ), which excludes the relevant presence of oxide domains near the edges of the flakes.

Photoelectrochemical Characterization. The LPEproduced $\mathrm{GaS}$ nanoflake dispersions were sprayed onto graphite paper, which acts as a non-photoactive current collector, to produce photoelectrode films. The PEC properties of the as-produced devices were evaluated in a three-electrode system (Figure $4 \mathrm{a}$ ) in different aqueous electrolytes: acidic $1 \mathrm{M}$ $\mathrm{H}_{2} \mathrm{SO}_{4}(\mathrm{pH}=0.5)$, near-neutral $1 \mathrm{M} \mathrm{Na}_{2} \mathrm{SO}_{4}(\mathrm{pH}=6)$, and alkaline $1 \mathrm{M} \mathrm{KOH}(\mathrm{pH}=14)$, investigating the response under $\mathrm{UV} /$ visible excitation wavelengths. To the best of our knowledge, the PEC properties of $\mathrm{GaS}$ flakes are currently unknown, although theoretical studies predicted their potential as water splitting photocatalysts. ${ }^{21,27}$ It is important to underline that the production of PEC-type devices is generally advantageous in terms of cost and ease of implementation compared to photodetectors based on isolated flakes. ${ }^{98-100}$ In principle, solution-processed $2 \mathrm{D}$ material films can also be deposited on interdigitated electrode to produce solid-state photodetectors. However, differently from solid-state photodetectors based on other solution-processed films of transition metal monochalcogenides (such as InSe), ${ }^{12,60}$ our attempts on photodetectors based on sprayed $\mathrm{GaS}$ flake films have shown poor device performance, i.e., responsivity lower than $0.4 \mathrm{~mA}$ $\mathrm{W}^{-1}$ under $275 \mathrm{~nm}$ illumination with an intensity of $1.3 \mathrm{~mW}$ $\mathrm{cm}^{-2}$ (see Optical Characterization in the Supporting Information, Figure S5). These results are ascribed to the low mobility of the GaS flakes $\left(\sim 0.1 \mathrm{~cm}^{2} \mathrm{~V}^{-1} \mathrm{~s}^{-1}\right),{ }^{61}$ which results in a highly resistive percolating network hindering the immediate exploitation of the peculiar optoelectronic proper-
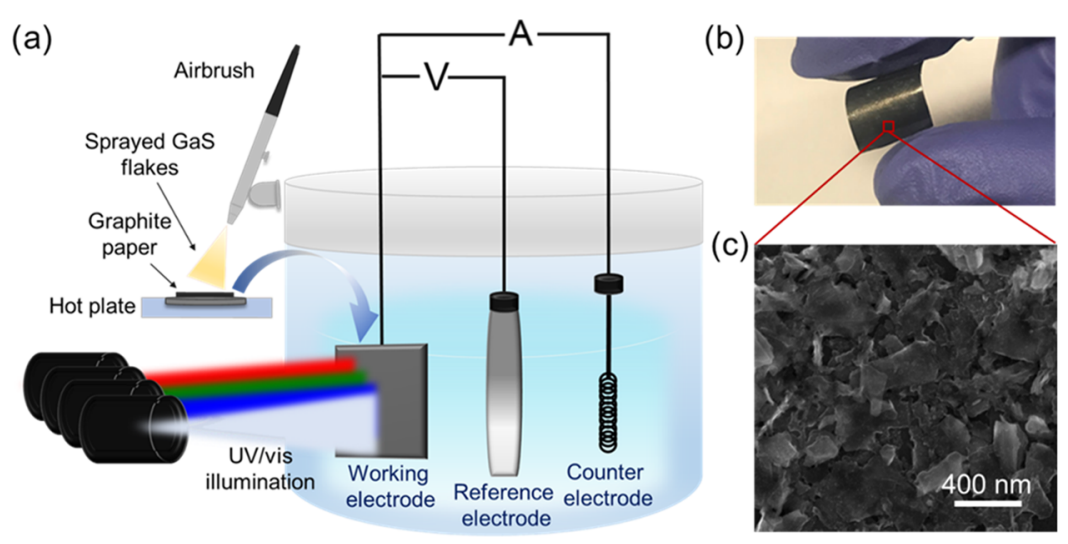

Figure 4. (a) Sketch of the experimental setup used for characterization of the PEC-type GaS photoelectrodes, which were produced by spray coating the $\mathrm{GaS}$ nanoflakes on a substrate of graphite paper, acting as the current collector. (b) Photograph of a flexible GaS photoelectrode. (c) Top-view SEM image of a GaS photoelectrode. 


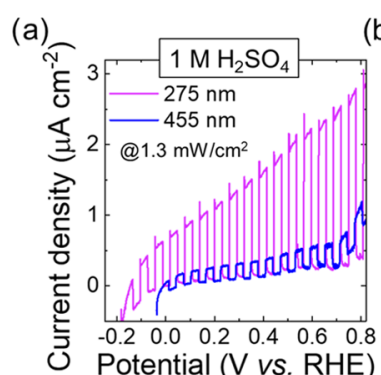

(d)

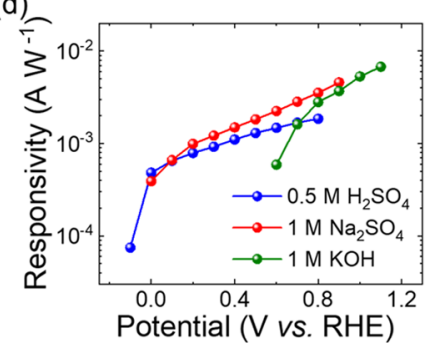

(c)

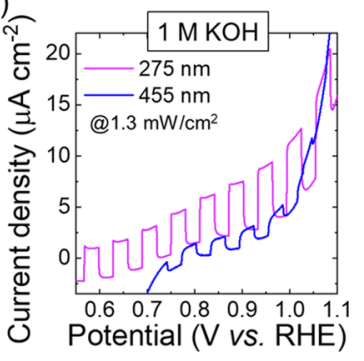

(e)

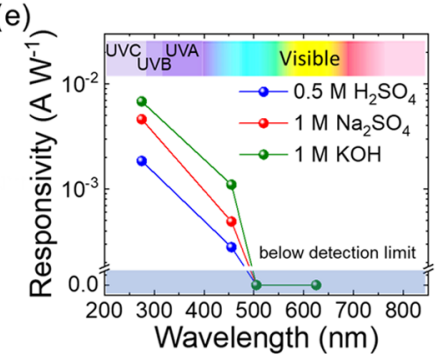

Figure 5. LSV scans of GaS PEC-type photodetectors in (a) $1 \mathrm{M} \mathrm{H}_{2} \mathrm{SO}_{4}$, (b) $1 \mathrm{M} \mathrm{Na}_{2} \mathrm{SO}_{4}$, and (c) $1 \mathrm{M} \mathrm{KOH}$ under UV (275 nm) and blue (455 $\mathrm{nm}$ ) illumination with an intensity of $1.3 \mathrm{~mW} \mathrm{~cm}^{-2}$. (d) Potential dependence of the responsivity of the GaS PEC-type photodetectors under 275 $\mathrm{nm}$ illumination with an intensity of $1.3 \mathrm{~mW} \mathrm{~cm}^{-2}$ in the three investigated aqueous media. For the device operating in $1 \mathrm{M} \mathrm{KOH}$, the potentials at which the device displayed significant negative dark current were excluded from the $x$-axis data range. (e) Wavelength dependence of the device responsivity under the same illumination intensity of $1.3 \mathrm{~mW} \mathrm{~cm}^{-2}$ in the three investigated aqueous media.

ties of the solution-processed $2 \mathrm{D}$ GaS. Figure $4 \mathrm{~b}$ reports a photograph of the GaS photoelectrode. Figures 4c and S6 show top-view SEM images of a GaS photoelectrode, which displays a porous film of flakes preferentially oriented with planes mostly parallel to the current collector.

The GaS photoelectrodes were evaluated as PEC-type photodetectors for four different illumination wavelengths in the UV/visible spectral range, namely, 275, 455, 505, and 625 $\mathrm{nm}$. It is noteworthy that only the illumination wavelength of $275 \mathrm{~nm}$ (hard UV-UVC region) corresponds to energy above the direct $E_{\mathrm{g}}$ estimated for the GaS flakes $(\sim 2.9 \mathrm{eV})$, while both 275 and $455 \mathrm{~nm}$ correspond to energy higher than the material indirect $E_{\mathrm{g}}(\sim 2.6 \mathrm{eV})$. Figure $5 \mathrm{a}-\mathrm{c}$ shows the anodic linear sweep voltammetry (LSV) measurements for the GaS photodetectors under chopped illumination (frequency $=0.33$ $\mathrm{Hz}$ ) at excitation wavelengths of 275 and $455 \mathrm{~nm}$ with an intensity of $1.3 \mathrm{~mW} \mathrm{~cm}^{-2}$ in the three investigated aqueous media, i.e., $1 \mathrm{M} \mathrm{H}_{2} \mathrm{SO}_{4}, 1 \mathrm{M} \mathrm{Na}_{2} \mathrm{SO}_{4}$, and $1 \mathrm{M} \mathrm{KOH}$. It is noteworthy that previous first-principles calculations predicted a photocatalytic activity of GaS flakes toward the OER. ${ }^{27}$ In detail, despite the dependence of the $E_{\mathrm{g}}$ on the number of layers, it was proven that both single- and few $(\leq 5)$-layer $\mathrm{GaS}$ flakes, as well as multi (>5)-layer $\mathrm{GaS}$ flakes, fulfill the fundamental requirements to carry out the OER, i.e., $E_{\mathrm{VBM}}<$ $E\left(\mathrm{O}_{2} / \mathrm{H}_{2} \mathrm{O}\right)$ for all of the $\mathrm{pH}$ conditions investigated in our work. ${ }^{27}$ Meanwhile, our preliminary cathodic LSV measurements did not show any photocathodic response of the GaS photoelectrodes, which were therefore analyzed only as photoanodes. In acidic media, the absence of the photocathodic response is attributed to the poor electrocatalytic activity of few-layer GaS flakes toward the HER (experimental reaction overpotential higher than $0.4 \mathrm{~V}) .^{28}$ In addition, in mild acidic and neutral or alkaline conditions, the energy offset between the $E_{\mathrm{CBM}}$ and $E\left(\mathrm{H}^{+} / \mathrm{H}_{2}\right)$ is significantly smaller than the one between $E_{\mathrm{VBM}}$ and $E\left(\mathrm{O}_{2} / \mathrm{H}_{2} \mathrm{O}\right) .{ }^{27}$ Therefore, the electronic structure of $2 \mathrm{D} \mathrm{GaS}$ promotes the OER in a PEC cell architecture without any charge extraction layers and/or cocatalysts, as in our case. It is noteworthy that an anodic PEC behavior similar to our $\mathrm{GaS}$ flakes has also been observed for $2 \mathrm{D} \mathrm{InSe}{ }^{67}$ for which an unsatisfactory electrocatalytic activity toward HER has been observed in both acidic and alkaline media. ${ }^{101}$ To avoid the electrochemically induced degradation of the photoelectrode, the applied potentials were limited to regions with dark current density substantially inferior to the detected photocurrents. Moreover, zero (or below-detection sensitivity) photoresponses were detected for the illumination wavelengths of 505 and $625 \mathrm{~nm}$. In all of the investigated media, the photoresponses of the photoanodes under UV light $(275 \mathrm{~nm})$ are significantly higher than those measured for blue light $(455 \mathrm{~nm})$, indicating a UV-selective photoresponse. Moreover, in $1 \mathrm{M} \mathrm{KOH}$, the photoanodes displayed negative dark currents above the open-circuit potential under illumination, i.e., at a potential between 0.3 and $0.8 \mathrm{~V}$ vs reversible hydrogen electrode (RHE). Since the chemical reactivity of the substrate was excluded for such conditions, the negative dark current at a potential inferior to $\sim 0.8 \mathrm{~V}$ vs $\mathrm{RHE}$ can be likely ascribed to corrosion effects involving $\mathrm{GaS}$ flakes.

Figure 5d reports the responsivity of the $\mathrm{GaS}$ photoanodes as a function of the applied potential in the three investigated media. The highest recorded responsivities for the $275 \mathrm{~nm}$ illumination are $1.8 \mathrm{~mA} \mathrm{~W}^{-1}$ for $1 \mathrm{M} \mathrm{H}_{2} \mathrm{SO}_{4}$ at $0.8 \mathrm{~V}$ vs RHE, $4.6 \mathrm{~mA} \mathrm{~W}^{-1}$ for $1 \mathrm{M} \mathrm{Na}_{2} \mathrm{SO}_{4}$ at $0.9 \mathrm{~V}$ vs RHE, and $6.8 \mathrm{~mA}$ $\mathrm{W}^{-1}$ for $1 \mathrm{M} \mathrm{KOH}$ at 1.1 . $\mathrm{V}$ vs RHE. Importantly, the highest photoresponse of $\mathrm{GaS}$ flakes has been observed in alkaline conditions, since the energy offset between $E_{\mathrm{VBM}}$ and $E\left(\mathrm{O}_{2} /\right.$ $\left.\mathrm{H}_{2} \mathrm{O}\right)$ is maximized compared to both the neutral and acidic media. ${ }^{27}$ Figure 5e shows the wavelength-dependent responsivity of the GaS photodetectors, indicating UV-selective light detection for UV-sensitive applications. These results agree with previous studies on solid-state photodetectors based on stoichiometric $\mathrm{GaS}$ flakes. ${ }^{22}$ Overall, these results suggest that the direct band gap transition is the main pathway driving the PEC activity of the devices. The low responsivity to blue light $(455 \mathrm{~nm})$ can be likely ascribed to either indirect band gap 
absorption or sub-band-gap states, which can also contribute to the photoresponse to blue light in solid-state photodetectors based on isolated GaS flakes. ${ }^{22}$ It is noteworthy that indirect band gap transitions also involve phonons, resulting in ineffective light absorption, especially in ultrathin films. ${ }^{102}$ Table S2 shows the comparison between the responsivity of our PEC-type $\mathrm{GaS}$ photodetectors and those of other solutionprocessed UV photodetectors based on 2D materials. Clearly, the combination of GaS flakes and the use of a PEC-type architecture is effective at overcoming the performance of several solid-state UV photodetectors based on solutionprocessed $2 \mathrm{D}$ materials reported in previous literature. ${ }^{103-107}$ In addition, the performances of our PEC-type GaS photodetectors can be one order of magnitude higher than those exhibited by their solid-state analogues based on $\mathrm{GaS}$ flake films deposited onto interdigitated electrodes (Figure S4). Lastly, the validation of the GaS-based PEC-type photodetectors can be useful for the design of PEC analytic systems that operate with low voltage sources, ${ }^{67,108,109}$ without recurring complex device manufacturing. For example, the detection of an analyte by means of PEC reactions can offer several benefits compared to electrochemical sensors. For example, PEC-type sensors can reduce the background signal down to the limit of lock-in detection noise when they operate in differential mode. ${ }^{110}$ Meanwhile, contrary to electrochemical sensors, they can eliminate the need for numerous recalibrations. ${ }^{111,112}$ Therefore, our validation of PEC-type GaS-based photodetectors with UV sensitivity may pave a new way for the realization of novel concepts of sensing devices.

The stability of $\mathrm{GaS}$ photodetectors was evaluated through subsequent LSV scans. As shown in Figure S7a, the GaS photoelectrodes show Raman spectra similar to the one measured for the as-produced GaS flakes, which means that the $\mathrm{GaS}$ flakes retain their starting structural properties during the PEC tests. The devices exhibit the most stable PEC performance in $1 \mathrm{M} \mathrm{KOH}$, showing a progressive responsivity stabilization over the first 10 LSV scans (Figure S7b). The initial degradation may be ascribed to the mechanical delamination induced by progressive gas evolution (i.e., oxygen evolution due to OER), as previously reported for similar architectures based on transition-metal monochalcogenides (e.g., $\mathrm{GaSe}^{9}$ and $\mathrm{GeSe}^{13}$ ). Prospectively, the use of polymeric (e.g., Nafion) $)^{13,113,114}$ or conductive (e.g., carbon nanotubes) binders, ${ }^{115-117}$ as well as the engineering of devices with effective charge-extracting layers and cocatalysts, ${ }^{118-120}$ could help to further stabilize the $\mathrm{GaS}$ photoelectrodes.

\section{CONCLUSIONS}

In summary, we presented the first PEC characterization of $2 \mathrm{D}$ $\mathrm{GaS}$ flakes produced through LPE methods in IPA. Our results provide novel insights into the fundamental PEC properties of 2D GaS flakes, which can be used to design and realize innovative PEC-type UV-selective photodetectors for medical diagnostics, air purification, chemical analysis (ozone sensing), and advanced optical communication systems. ${ }^{121-125}$ In particular, LPE-produced GaS flakes can be easily deposited through printing techniques to produce solution-processed photocatalytic films on graphite paper, the latter acting as a current collector of the resulting photoelectrodes. Our PEC characterizations indicate that solution-processed 2D GaSbased PEC-type photodetectors outperform the corresponding solid-state photodetectors. In fact, the $2 \mathrm{D}$ morphology of the $\mathrm{GaS}$ flakes intrinsically minimizes the distance between the photogenerated charges and the surface area at which the redox reactions occur, limiting electron-hole recombination losses compared to the case of standard solid-state configurations made of the same photoactive films. Therefore, our PEC-type GaS photodetectors display a relevant UVselective PEC photoresponse, attaining responsivities of 1.8 $\mathrm{mA} \mathrm{W}^{-1}$ in $1 \mathrm{M} \mathrm{H}_{2} \mathrm{SO}_{4}$ (at $0.8 \mathrm{~V}$ vs RHE), $4.6 \mathrm{~mA} \mathrm{~W}^{-1}$ in $1 \mathrm{M}$ $\mathrm{Na}_{2} \mathrm{SO}_{4}$ (at $0.9 \mathrm{~V}$ vs RHE), and $6.8 \mathrm{~mA} \mathrm{~W}^{-1}$ in $1 \mathrm{M} \mathrm{KOH} \mathrm{(at}$ 1.1. V vs RHE) under $275 \mathrm{~nm}$ illumination wavelength with an intensity of $1.3 \mathrm{~mW} \mathrm{~cm} \mathrm{~cm}^{-2}$. Beyond the photodetector application, GaS-based PEC-type devices can find application in tandem solar PEC cells in combination with other visiblesensitive low-band-gap materials, including transition-metal monochalcogenides recently established for PEC solar energy conversion applications. ${ }^{126-128}$

\section{ASSOCIATED CONTENT}

\section{Supporting Information}

The Supporting Information is available free of charge at https://pubs.acs.org/doi/10.1021/acs.jpcc.1c03597.

Methods, AFM analysis, SEM-EDS analysis, Raman spectroscopy analysis, XPS characterization, characterization of the solid-state photodetectors, and performance comparison with the literature (PDF).

\section{AUTHOR INFORMATION}

\section{Corresponding Authors}

Sebastiano Bellani - BeDimensional Spa., 16163 Genova, Italy; Graphene Labs, Istituto Italiano di Tecnologia, 16163 Genova, Italy; Email: s.bellani@bedimensional.it

Francesco Bonaccorso - BeDimensional Spa., 16163 Genova, Italy; Graphene Labs, Istituto Italiano di Tecnologia, 16163 Genova, Italy; 이이이.org/0000-0001-7238-9420; Email: f.bonaccorso@bedimensional.it

\section{Authors}

Marilena I. Zappia - BeDimensional Spa., 16163 Genova, Italy; Department of Physics, University of Calabria, 87036 Rende, CS, Italy

Gabriele Bianca - Graphene Labs, Istituto Italiano di Tecnologia, 16163 Genova, Italy; Dipartimento di Chimica e Chimica Industriale, Università degli Studi di Genova, 16146 Genoa, Italy

Nicola Curreli - Functional Nanosystems, Istituto Italiano di Tecnologia (IIT), 16163 Genova, Italy

Zdeněk Sofer - Department of Inorganic Chemistry, University of Chemistry and Technology Prague, 16628 Prague 6, Czech Republic; (1) orcid.org/0000-0002-13914448

Michele Serri - Graphene Labs, Istituto Italiano di Tecnologia, 16163 Genova, Italy; 이이.org/0000-00026018-5284

Leyla Najafi - BeDimensional Spa., 16163 Genova, Italy; Graphene Labs, Istituto Italiano di Tecnologia, 16163 Genova, Italy

Marco Piccinni - Graphene Labs, Istituto Italiano di Tecnologia, 16163 Genova, Italy; Dipartimento di Chimica e Chimica Industriale, Università degli Studi di Genova, 16146 Genoa, Italy

Reinier Oropesa-Nuñez - BeDimensional Spa., 16163 Genova, Italy; Department of Material Science and 
Engineering, Uppsala University, 75121 Uppsala, Sweden; (1) orcid.org/0000-0002-9551-6565

Petr Marvan - Department of Inorganic Chemistry, University of Chemistry and Technology Prague, 16628 Prague 6, Czech Republic

Vittorio Pellegrini - BeDimensional Spa., 16163 Genova, Italy; Graphene Labs, Istituto Italiano di Tecnologia, 16163 Genova, Italy

Ilka Kriegel - Functional Nanosystems, Istituto Italiano di Tecnologia (IIT), 16163 Genova, Italy; 이이.org/00000002-0221-3769

Mirko Prato - Materials Characterization Facility, Istituto Italiano di Tecnologia, Genova 16163, Italy; o orcid.org/ 0000-0002-2188-8059

Anna Cupolillo - Department of Physics, University of Calabria, 87036 Rende, CS, Italy

Complete contact information is available at:

https://pubs.acs.org/10.1021/acs.jpcc.1c03597

\section{Author Contributions}

M.I.Z. and G.B. contributed equally to this work. The manuscript was written through contributions of all authors. All authors have given approval to the final version of the manuscript.

Notes

The authors declare no competing financial interest.

\section{ACKNOWLEDGMENTS}

This project has received funding from the European Union's Horizon 2020 European Research Council under grant agreement no. [850875] (Light-DYNAMO) and from the European Union's Horizon 2020 Research and Innovation programme under grant agreement no. [101017821] (LIGHTCAP) and grant agreement no. 881603-GrapheneCore3, the MSCA-ITN ULTIMATE project under grant agreement no. 813036, and the Bilateral project GINSENG between NSFC (China) and MAECI (Italy) (2018-2020) by the Natural Science Foundation of Shandong Province (ZR2019QEM009). This project was supported by the Czech Science Foundation (GACR No. 20-16124J). P.M. was supported by specific university research grant no. A2_FCHT_2020_055. M.I.Z. received funding from PON Research an $\bar{d}$ Innovation 2014-2020 (CUP H25D18000230006) by the Italian Ministry of University and Research. We thank the Materials Characterization Facility-Istituto Italiano di Tecnologia-for the support in XRD data acquisition/analysis and Electron Microscopy facility-Istituto Italiano di Tecnologia-for the support in TEM data acquisition.

\section{ABBREVIATIONS}

2D, two-dimensional; AFM, atomic force microscopy; EDS, energy-dispersive X-ray spectroscopy; $E_{g}$, optical band gap; Ga, gallium sulfide; IPA, 2-propanol; LED, light-emitting diode; LPE, liquid-phase exfoliation; HER, hydrogen evolution reaction; NMP, N-methyl-2-pyrrolidone; $R$, diffusive reflectance; RHE, reversible hydrogen electrode; OER, oxygen evolution reaction; PEC, photoelectrochemical; SEM, scanning electron microscopy; TEM, transmission electron microscopy; $\mathrm{UV}$, ultraviolet; XPS, X-ray photoelectron spectroscopy; XRD, $\mathrm{X}$-ray diffraction

\section{REFERENCES}

(1) Li, X.; Tao, L.; Chen, Z.; Fang, H.; Li, X.; Wang, X.; Xu, J.-B.; Zhu, H. Graphene and Related Two-Dimensional Materials: Structure-Property Relationships for Electronics and Optoelectronics. Appl. Phys. Rev. 2017, 4, No. 021306.

(2) Xu, K.; Yin, L.; Huang, Y.; Shifa, T. A.; Chu, J.; Wang, F.; Cheng, R.; Wang, Z.; He, J. Synthesis, Properties and Applications of 2D Layered M III X VI ( $\mathrm{M}=\mathrm{Ga}$, In; X = S, Se, Te) Materials. Nanoscale 2016, 8, 16802-16818.

(3) Cai, H.; Gu, Y.; Lin, Y.-C.; Yu, Y.; Geohegan, D. B.; Xiao, K. Synthesis and Emerging Properties of 2D Layered III-VI Metal Chalcogenides. Appl. Phys. Rev. 2019, 6, No. 041312.

(4) Terhell, J. C. J. M. Polytypism in the III-VI Layer Compounds. Prog. Cryst. Growth Charact. 1983, 7, 55-110.

(5) Kuhn, A.; Chevy, A.; Chevalier, R. Refinement of the $2 \mathrm{H} \mathrm{GaS} \beta$ Type. Acta Crystallogr., Sect. B: Struct. Crystallogr. Cryst. Chem. 1976, 32, 983-984.

(6) Kuhn, A.; Bourdon, A.; Rigoult, J.; Rimsky, A. Charge-Density Analysis of GaS. Phys. Rev. B 1982, 25, 4081-4088.

(7) Kokh, K. A.; Andreev, Y. M.; Svetlichnyi, V. A.; Lanskii, G. V.; Kokh, A. E. Growth of GaSe and GaS Single Crystals. Cryst. Res. Technol. 2011, 46, 327-330.

(8) Brudnyi, V. N.; Sarkisov, S. Y.; Kosobutsky, A. V. Electronic Properties of GaSe, InSe, GaS and GaTe Layered Semiconductors: Charge Neutrality Level and Interface Barrier Heights. Semicond. Sci. Technol. 2015, 30, No. 115019.

(9) Zappia, M. I.; Bianca, G.; Bellani, S.; Serri, M.; Najafi, L.; Oropesa-Nuñez, R.; Martín-García, B.; Bouša, D.; Sedmidubský, D.; Pellegrini, V.; et al. Solution-Processed GaSe Nanoflake-Based Films for Photoelectrochemical Water Splitting and PhotoelectrochemicalType Photodetectors. Adv. Funct. Mater. 2020, 30, No. 1909572.

(10) Curreli, N.; Serri, M.; Zappia, M. I.; Spirito, D.; Bianca, G.; Buha, J.; Najafi, L.; Sofer, Z.; Krahne, R.; Pellegrini, V.; Bonaccorso, F.; et al. Liquid-Phase Exfoliated Gallium Selenide for Light-Driven Thin-Film Transistors. Adv. Electron. Mater. 2021, 7, No. 2001080.

(11) Petroni, E.; Lago, E.; Bellani, S.; Boukhvalov, D. W.; Politano, A.; Gürbulak, B.; Duman, S.; Prato, M.; Gentiluomo, S.; OropesaNuñez, R.; et al. Liquid-Phase Exfoliated Indium-Selenide Flakes and Their Application in Hydrogen Evolution Reaction. Small 2018, 14, No. 1800749.

(12) Curreli, N.; Serri, M.; Spirito, D.; Lago, E.; Petroni, E.; MartínGarcía, B.; Politano, A.; Gürbulak, B.; Duman, S.; Krahne, R.; et al. Liquid Phase Exfoliated Indium Selenide Based Highly Sensitive Photodetectors. Adv. Funct. Mater. 2020, 30, No. 1908427.

(13) Bianca, G.; Zappia, M. I.; Bellani, S.; Sofer, Z.; Serri, M.; Najafi, L.; Oropesa-Nuñez, R.; Martín-García, B.; Hartman, T.; Leoncino, L.; et al. Liquid-Phase Exfoliated GeSe Nanoflakes for Photoelectrochemical-Type Photodetectors and Photoelectrochemical Water Splitting. ACS Appl. Mater. Interfaces 2020, 12, 48598-48613.

(14) Gonzalez, J. M.; Oleynik, I. I. Layer-Dependent Properties of $\mathrm{SnS}_{2}$ and $\mathrm{SnSe}_{2}$ Two-Dimensional Materials. Phys. Rev. B 2016, 94, No. 125443.

(15) Ho, C. H.; Lin, S. L. Optical Properties of the Interband Transitions of Layered Gallium Sulfide. J. Appl. Phys. 2006, 100, No. 083508.

(16) Ho, C. H.; Huang, K. W. Visible Luminescence and Structural Property of GaSe1-xSx $(0 \leq \mathrm{x} \leq 1)$ Series Layered Crystals. Solid State Commun. 2005, 136, 591-594.

(17) Aulich, E.; Brebner, J. L.; Mooser, E. Indirect Energy Gap in GaSe and GaS. Phys. Status Solidi B 1969, 31, 129-131.

(18) Cingolani, A.; Minafra, A.; Tantalo, P.; Paorici, C. Edge Emission in GaSe and GaS. Phys. Status Solidi A 1971, 4, K83-K85.

(19) Chen, H.; Li, Y.; Huang, L.; Li, J. Intrinsic Defects in Gallium Sulfide Monolayer: A First-Principles Study. RSC Adv. 2015, 5, 50883-50889.

(20) Jung, C. S.; Shojaei, F.; Park, K.; Oh, J. Y.; Im, H. S.; Jang, D. M.; Park, J.; Kang, H. S. Red-to-Ultraviolet Emission Tuning of TwoDimensional Gallium Sulfide/Selenide. ACS Nano 2015, 9, 95859593. 
(21) Zhuang, H. L.; Hennig, R. G. Single-Layer Group-III Monochalcogenide Photocatalysts for Water Splitting. Chem. Mater. 2013, 25, 3232-3238.

(22) Lu, Y.; Chen, J.; Chen, T.; Shu, Y.; Chang, R.; Sheng, Y.; Shautsova, V.; Mkhize, N.; Holdway, P.; Bhaskaran, H.; et al. Controlling Defects in Continuous 2D GaS Films for HighPerformance Wavelength-Tunable UV-Discriminating Photodetectors. Adv. Mater. 2020, 32, No. 1906958.

(23) Hu, P.; Wang, L.; Yoon, M.; Zhang, J.; Feng, W.; Wang, X.; Wen, Z.; Idrobo, J. C.; Miyamoto, Y.; Geohegan, D. B.; et al. Highly Responsive Ultrathin GaS Nanosheet Photodetectors on Rigid and Flexible Substrates. Nano Lett. 2013, 13, 1649-1654.

(24) Ho, C.-H.; Hsieh, M.-H.; Wu, C.-C. Photoconductance and Photoresponse of Layer Compound Photodetectors in the UV-Visible Region. Rev. Sci. Instrum. 2006, 77, No. 113102.

(25) Peng, Q.; Guo, Z.; Sa, B.; Zhou, J.; Sun, Z. New Gallium Chalcogenides/Arsenene van Der Waals Heterostructures Promising for Photocatalytic Water Splitting. Int. J. Hydrogen Energy 2018, 43, No. 15995.

(26) Tsoeu, S. E.; Opoku, F.; Govender, P. P. Tuning the Electronic, Optical and Structural Properties of $\mathrm{GaS} / \mathrm{C} 2 \mathrm{~N}$ van Der Waals Heterostructure for Photovoltaic Application: First-Principle Calculations. SN Appl. Sci. 2020, 2, No. 341.

(27) Cui, Y.; Peng, L.; Sun, L.; Qian, Q.; Huang, Y. TwoDimensional Few-Layer Group-III Metal Monochalcogenides as Effective Photocatalysts for Overall Water Splitting in the Visible Range. J. Mater. Chem. A 2018, 6, 22768-22777.

(28) Harvey, A.; Backes, C.; Gholamvand, Z.; Hanlon, D.; McAteer, D.; Nerl, H. C.; McGuire, E.; Seral-Ascaso, A.; Ramasse, Q. M.; McEvoy, N.; et al. Preparation of Gallium Sulfide Nanosheets by Liquid Exfoliation and Their Application As Hydrogen Evolution Catalysts. Chem. Mater. 2015, 27, 3483-3493.

(29) Mishra, P.; Singh, D.; Sonvane, Y.; Ahuja, R. Enhancement of Hydrogen Storage Capacity on Co-Functionalized GaS Monolayer under External Electric Field. Int. J. Hydrogen Energy 2020, 45, 12384-12393.

(30) Zhang, C. J.; Park, S.-H.; Ronan, O.; Harvey, A.; Seral-Ascaso, A.; Lin, Z.; McEvoy, N.; Boland, C. S.; Berner, N. C.; Duesberg, G. S.; et al. Enabling Flexible Heterostructures for Li-Ion Battery Anodes Based on Nanotube and Liquid-Phase Exfoliated 2D Gallium Chalcogenide Nanosheet Colloidal Solutions. Small 2017, 13, No. 1701677.

(31) Meng, X.; He, K.; Su, D.; Zhang, X.; Sun, C.; Ren, Y.; Wang, H.-H.; Weng, W.; Trahey, L.; Canlas, C. P.; et al. Gallium SulfideSingle-Walled Carbon Nanotube Composites: High-Performance Anodes for Lithium-Ion Batteries. Adv. Funct. Mater. 2014, 24, $5435-5442$.

(32) Yang, S.; Li, Y.; Wang, X.; Huo, N.; Xia, J.-B.; Li, S.-S.; Li, J. High Performance Few-Layer GaS Photodetector and Its Unique Photo-Response in Different Gas Environments. Nanoscale 2014, 6, 2582-2587.

(33) Marvan, P.; Mazánek, V.; Sofer, Z. Shear-Force Exfoliation of Indium and Gallium Chalcogenides for Selective Gas Sensing Applications. Nanoscale 2019, 11, 4310-4317.

(34) Singh, D.; Panda, P. K.; Mishra, Y. K.; Ahuja, R. Van Der Waals Induced Molecular Recognition of Canonical DNA Nucleobases on a 2D GaS Monolayer. Phys. Chem. Chem. Phys. 2020, 22, 6706-6715.

(35) Kato, K.; Umemura, N. Sellmeier Equations for GaS and GaSe and Their Applications to the Nonlinear Optics in GaS_xSe_1-x. Opt. Lett. 2011, 36, No. 746.

(36) Allakhverdiev, K.; Ismailov, F.; Kador, L.; Braun, M. SecondHarmonic Generation in GaS Crystals. Solid State Commun. 1997, 104, 1-3.

(37) Balendhran, S.; Walia, S.; Nili, H.; Sriram, S.; Bhaskaran, M. Elemental Analogues of Graphene: Silicene, Germanene, Stanene, and Phosphorene. Small 2015, 11, 640-652.

(38) Molle, A.; Grazianetti, C.; Chiappe, D.; Cinquanta, E.; Cianci, E.; Tallarida, G.; Fanciulli, M. Hindering the Oxidation of Silicene with Non-Reactive Encapsulation. Adv. Funct. Mater. 2013, 23, 43404344.

(39) Ye, F.; Lee, J.; Hu, J.; Mao, Z.; Wei, J.; Feng, P. X. L. Environmental Instability and Degradation of Single- and Few-Layer WTe 2 Nanosheets in Ambient Conditions. Small 2016, 12, 58025808.

(40) Jaegermann, W.; Schmeisser, D. Reactivity of Layer Type Transition Metal Chalcogenides towards Oxidation. Surf. Sci. 1986, $165,143-160$.

(41) Favron, A.; Gaufrès, E.; Fossard, F.; Phaneuf-Laheureux, A. L.; Tang, N. Y. W.; Lévesque, P. L.; Loiseau, A.; Leonelli, R.; Francoeur, S.; Martel, R. Photooxidation and Quantum Confinement Effects in Exfoliated Black Phosphorus. Nat. Mater. 2015, 14, 826-832.

(42) Walia, S.; Sabri, Y.; Ahmed, T.; Field, M. R.; Ramanathan, R.; Arash, A.; Bhargava, S. K.; Sriram, S.; Bhaskaran, M.; Bansal, V.; et al. Defining the Role of Humidity in the Ambient Degradation of FewLayer Black Phosphorus. 2D Mater. 2016, 4, No. 015025.

(43) Zhou, Q.; Chen, Q.; Tong, Y.; Wang, J. Light-Induced Ambient Degradation of Few-Layer Black Phosphorus: Mechanism and Protection. Angew. Chem., Int. Ed. 2016, 55, 11437-11441.

(44) Beechem, T. E.; Kowalski, B. M.; Brumbach, M. T.; McDonald, A. E.; Spataru, C. D.; Howell, S. W.; Ohta, T.; Pask, J. A.; Kalugin, N. G. Oxidation of Ultrathin GaSe. Appl. Phys. Lett. 2015, 107, No. 173103.

(45) Bergeron, A.; Ibrahim, J.; Leonelli, R.; Francoeur, S. Oxidation Dynamics of Ultrathin GaSe Probed through Raman Spectroscopy. Appl. Phys. Lett. 2017, 110, No. 241901.

(46) Zhao, Q.; Frisenda, R.; Gant, P.; Perez de Lara, D.; Munuera, C.; Garcia-Hernandez, M.; Niu, Y.; Wang, T.; Jie, W.; CastellanosGomez, A. Toward Air Stability of Thin GaSe Devices: Avoiding Environmental and Laser-Induced Degradation by Encapsulation. Adv. Funct. Mater. 2018, 28, No. 1805304.

(47) Shi, L.; Li, Q.; Ouyang, Y.; Wang, J. Effect of Illumination and Se Vacancies on Fast Oxidation of Ultrathin Gallium Selenide. Nanoscale 2018, 10, 12180-12186.

(48) Guo, Y.; Zhou, S.; Bai, Y.; Zhao, J. Oxidation Resistance of Monolayer Group-IV Monochalcogenides. ACS Appl. Mater. Interfaces 2017, 9, 12013-12020.

(49) Yang, C.; Lu, P.; Huang, W.; Chen, J. Mechanical Stabilities and Nonlinear Properties of Monolayer Gallium Sulfide under Tension. Superlattices Microstruct. 2015, 80, 80-90.

(50) Ma, Y.; Dai, Y.; Guo, M.; Yu, L.; Huang, B. Tunable Electronic and Dielectric Behavior of GaS and GaSe Monolayers. Phys. Chem. Chem. Phys. 2013, 15, 7098.

(51) Guo, Y.; Zhou, S.; Bai, Y.; Zhao, J. Defects and Oxidation of Group-III Monochalcogenide Monolayers. J. Chem. Phys. 2017, 147, No. 104709.

(52) Yi, M.; Shen, Z. A Review on Mechanical Exfoliation for the Scalable Production of Graphene. J. Mater. Chem. A 2015, 3, 1170011715 .

(53) Backes, C.; Abdelkader, A. M.; Alonso, C.; Andrieux-Ledier, A.; Arenal, R.; Azpeitia, J.; Balakrishnan, N.; Banszerus, L.; Barjon, J.; Bartali, R.; et al. Production and Processing of Graphene and Related Materials. 2D Mater. 2020, 7, No. 22001.

(54) Bonaccorso, F.; Bartolotta, A.; Coleman, J. N.; Backes, C. 2DCrystal-Based Functional Inks. Adv. Mater. 2016, 28, 6136-6166.

(55) Coleman, J. N.; Lotya, M.; O’Neill, A.; Bergin, S. D.; King, P. J.; Khan, U.; Young, K.; Gaucher, A.; De, S.; Smith, R. J.; Shvets, I. V.; et al. Two-Dimensional Nanosheets Produced by Liquid Exfoliation of Layered Materials. Science 2011, 331, 568-571.

(56) Bellani, S.; Petroni, E.; Del Rio Castillo, A. E.; Curreli, N.; Martín-García, B.; Oropesa-Nuñez, R.; Prato, M.; Bonaccorso, F. Scalable Production of Graphene Inks via Wet-Jet Milling Exfoliation for Screen-Printed Micro-Supercapacitors. Adv. Funct. Mater. 2019, 29, No. 1807659.

(57) Tsikritzis, D.; Rogdakis, K.; Chatzimanolis, K.; Petrović, M.; Tzoganakis, N.; Najafi, L.; Martín-García, B.; Oropesa-Nuñez, R.; Bellani, S.; Del Rio Castillo, A. E.; et al. A Two-Fold Engineering 
Approach Based on Bi 2 Te 3 Flakes towards Efficient and Stable Inverted Perovskite Solar Cells. Mater. Adv. 2020, 1, 450-462.

(58) Najafi, L.; Bellani, S.; Oropesa-nuñez, R.; Brescia, R.; Prato, M.; Pasquale, L.; Demirci, C.; Drago, F.; Martín-garcía, B.; Luxa, J.; et al. Microwave-Induced Structural Engineering and Pt Trapping in 6RTaS2 for Hydrogen Evolution. Small 2020, 16, No. 2003372.

(59) Torrisi, F.; Coleman, J. N. Electrifying Inks with 2D Materials. Nat. Nanotechnol. 2014, 9, 738-739.

(60) Kang, J.; Wells, S. A.; Sangwan, V. K.; Lam, D.; Liu, X.; Luxa, J.; Sofer, Z.; Hersam, M. C. Solution-Based Processing of Optoelectronically Active Indium Selenide. Adv. Mater. 2018, 30, No. 1802990.

(61) Late, D. J.; Liu, B.; Luo, J.; Yan, A.; Matte, H. S. S. R.; Grayson, M.; Rao, C. N. R.; Dravid, V. P. GaS and GaSe Ultrathin Layer Transistors. Adv. Mater. 2012, 24, 3549-3554.

(62) Feng, W.; Zheng, W.; Cao, W.; Hu, P. Back Gated Multilayer InSe Transistors with Enhanced Carrier Mobilities via the Suppression of Carrier Scattering from a Dielectric Interface. Adv. Mater. 2014, 26, 6587-6593.

(63) Bandurin, D. A.; Tyurnina, A. V.; Yu, G. L.; Mishchenko, A.; Zólyomi, V.; Morozov, S. V.; Kumar, R. K.; Gorbachev, R. V.; Kudrynskyi, Z. R.; Pezzini, S.; et al. High Electron Mobility, Quantum Hall Effect and Anomalous Optical Response in Atomically Thin InSe. Nat. Nanotechnol. 2017, 12, 223-227.

(64) Chowdhury, C.; Karmakar, S.; Datta, A. Monolayer Group IVVI Monochalcogenides: Low-Dimensional Materials for Photocatalytic Water Splitting. J. Phys. Chem. C 2017, 121, 7615-7624.

(65) Lv, X.; Wei, W.; Sun, Q.; Li, F.; Huang, B.; Dai, Y. TwoDimensional Germanium Monochalcogenides for Photocatalytic Water Splitting with High Carrier Mobility. Appl. Catal., B 2017, 217, 275-284.

(66) Ye, Y.; Guo, Q.; Liu, X.; Liu, C.; Wang, J.; Liu, Y.; Qiu, J. TwoDimensional GeSe as an Isostructural and Isoelectronic Analogue of Phosphorene: Sonication-Assisted Synthesis, Chemical Stability, and Optical Properties. Chem. Mater. 2017, 29, 8361-8368.

(67) Li, Z.; Qiao, H.; Guo, Z.; Ren, X.; Huang, Z.; Qi, X.; Dhanabalan, S. C.; Ponraj, J. S.; Zhang, D.; Li, J.; Zhao, J.; Zhong, J.; Zhang, H. High-Performance Photo-Electrochemical Photodetector Based on Liquid-Exfoliated Few-Layered InSe Nanosheets with Enhanced Stability. Adv. Funct. Mater. 2018, 28, No. 1705237.

(68) Wickramaratne, D.; Zahid, F.; Lake, R. K. Electronic and Thermoelectric Properties of van Der Waals Materials with RingShaped Valence Bands. J. Appl. Phys. 2015, 118, No. 075101.

(69) Faraji, M.; Yousefi, M.; Yousefzadeh, S.; Zirak, M.; Naseri, N.; Jeon, T. H.; Choi, W.; Moshfegh, A. Z. Two-Dimensional Materials in Semiconductor Photoelectrocatalytic Systems for Water Splitting. Energy Environ. Sci. 2019, 12, 59-95.

(70) Li, Y.; Li, Y.-L.; Sa, B.; Ahuja, R. Review of Two-Dimensional Materials for Photocatalytic Water Splitting from a Theoretical Perspective. Catal. Sci. Technol. 2017, 7, 545-559.

(71) Su, T.; Shao, Q.; Qin, Z.; Guo, Z.; Wu, Z. Role of Interfaces in Two-Dimensional Photocatalyst for Water Splitting. ACS Catal. 2018, 8, 2253-2276.

(72) Gan, X.; Lei, D.; Wong, K. Y. Two-Dimensional Layered Nanomaterials for Visible-Light-Driven Photocatalytic Water Splitting. Mater. Today Energy 2018, 352-367.

(73) Luo, B.; Liu, G.; Wang, L. Recent Advances in 2D Materials for Photocatalysis. Nanoscale 2016, 8, 6904-6920.

(74) Hu, S.; Zhu, M. Ultrathin Two-Dimensional Semiconductors for Photocatalysis in Energy and Environment Applications. ChemCatChem 2019, 11, 6147-6165.

(75) Haynes, W. M. CRC Handbook Chemistry and Physics; CRC Press, 2016.

(76) Najafi, L.; Bellani, S.; Martín-García, B.; Oropesa-Nunez, R.; Del Rio Castillo, A. E.; Prato, M.; Moreels, I.; Bonaccorso, F. Solution-Processed Hybrid Graphene Flake/2H-MoS2 Quantum Dot Heterostructures for Efficient Electrochemical Hydrogen Evolution. Chem. Mater. 2017, 29, 5782-5786.

(77) Najafi, L.; Bellani, S.; Oropesa-Nuñez, R.; Ansaldo, A.; Prato, M.; Del Rio Castillo, A. E.; Bonaccorso, F. Doped-MoSe2
Nanoflakes/3d Metal Oxide-Hydr(Oxy)Oxides Hybrid Catalysts for PH-Universal Electrochemical Hydrogen Evolution Reaction. Adv. Energy Mater. 2018, 8, No. 1801764.

(78) Najafi, L.; Romano, V.; Oropesa-Nuñez, R.; Prato, M.; Lauciello, S.; D'Angelo, G.; Bellani, S.; Bonaccorso, F. Hybrid Organic/Inorganic Photocathodes Based on WS2 Flakes as Hole Transporting Layer Material. Small Struct. 2021, 2, No. 2000098.

(79) Bellani, S.; Martín-García, B.; Oropesa-Nuñez, R.; Romano, V.; Najafi, L.; Demirci, C.; Prato, M.; Del Rio Castillo, A. E.; Marasco, L.; Mantero, E.; et al. "Ion Sliding" on Graphene: A Novel Concept to Boost Supercapacitor Performance. Nanoscale Horiz. 2019, 4, 10771091.

(80) Ansaldo, A.; Bondavalli, P.; Bellani, S.; Del Rio Castillo, A. E.; Prato, M.; Pellegrini, V.; Pognon, G.; Bonaccorso, F. High-Power Graphene-Carbon Nanotube Hybrid Supercapacitors. ChemNanoMat 2017, 3, 436-446.

(81) Jawaid, A.; Nepal, D.; Park, K.; Jespersen, M.; Qualley, A.; Mirau, P.; Drummy, L. F.; Vaia, R. A. Mechanism for Liquid Phase Exfoliation of MoS2. Chem. Mater. 2016, 28, 337-348.

(82) Shen, G.; Chen, D.; Chen, P.-C.; Zhou, C. Vapor-Solid Growth of One-Dimensional Layer-Structured Gallium Sulfide Nanostructures. ACS Nano 2009, 3, 1115-1120.

(83) Preparation and Crystal Growth of Materials with Layered Structures; Lieth, R. M. A., Ed.; Springer: Dordrecht, Netherlands, 1977.

(84) Late, D. J.; Liu, B.; Matte, H. S. S. R.; Rao, C. N. R.; Dravid, V. P. Rapid Characterization of Ultrathin Layers of Chalcogenides on SiO2/Si Substrates. Adv. Funct. Mater. 2012, 22, 1894-1905.

(85) Hernandez, Y.; Nicolosi, V.; Lotya, M.; Blighe, F. M.; Sun, Z.; De, S.; McGovern, I. T.; Holland, B.; Byrne, M.; Gun'Ko, Y. K.; et al. High-Yield Production of Graphene by Liquid-Phase Exfoliation of Graphite. Nat. Nanotechnol. 2008, 3, 563-568.

(86) Yang, L.; Miklavcic, S. J. Revised Kubelka-Munk Theory III A General Theory of Light Propagation in Scattering and Absorptive Media. J. Opt. Soc. Am. A 2005, 22, No. 1866.

(87) Vargas, W. E.; Niklasson, G. A. Applicability Conditions of the Kubelka-Munk Theory. Appl. Opt. 1997, 36, No. 5580.

(88) Najafi, L.; Taheri, B.; Martín-García, B.; Bellani, S.; Di Girolamo, D.; Agresti, A.; Oropesa-Nuñez, R.; Pescetelli, S.; Vesce, L.; Calabrò, E.; et al. MoS2 Quantum Dot/Graphene Hybrids for Advanced Interface Engineering of a $\mathrm{CH} 3 \mathrm{NH} 3 \mathrm{PbI} 3$ Perovskite Solar Cell with an Efficiency of over 20\%. ACS Nano 2018, 12, 1073610754.

(89) Antunez, P. D.; Torelli, D. A.; Yang, F.; Rabuffetti, F. A.; Lewis, N. S.; Brutchey, R. L. Low Temperature Solution-Phase Deposition of SnS Thin Films. Chem. Mater. 2014, 26, 5444-5446.

(90) Patel, M.; Chavda, A.; Mukhopadhyay, I.; Kim, J.; Ray, A. Nanostructured SnS with Inherent Anisotropic Optical Properties for High Photoactivity. Nanoscale 2016, 8, 2293-2303.

(91) Materials Data on GaS by Materials Project. https://www.osti. gov/dataexplorer/biblio/dataset/1200464 (accessed June 4, 2021).

(92) Gasanly, N. M.; Aydınlı, A.; Özkan, H.; Kocabaş, C. Temperature Dependence of the First-Order Raman Scattering in GaS Layered Crystals. Solid State Commun. 2000, 116, 147-151.

(93) Irwin, J. C.; Hoff, R. M.; Clayman, B. P.; Bromley, R. A. Long Wavelength Lattice Vibrations in $\mathrm{GaS}$ and GaSe. Solid State Commun. 1973, 13, 1531-1536.

(94) Jastrzebski, C.; Olkowska, K.; Jastrzebski, D. J.; Wierzbicki, M.; Gebicki, W.; Podsiadlo, S. Raman Scattering Studies on Very Thin Layers of Gallium Sulfide $(\mathrm{GaS})$ as a Function of Sample Thickness and Temperature. J. Phys.: Condens. Matter 2019, 31, No. 075303.

(95) Demirci, S.; Avazlı, N.; Durgun, E.; Cahangirov, S. Structural and Electronic Properties of Monolayer Group III Monochalcogenides. Phys. Rev. B 2017, 95, No. 115409.

(96) Bellani, S.; Najafi, L.; Capasso, A.; Del Rio Castillo, A. E.; Antognazza, M. R.; Bonaccorso, F. Few-Layer MoS 2 Flakes as a Hole-Selective Layer for Solution-Processed Hybrid Organic Hydrogen-Evolving Photocathodes. J. Mater. Chem. A 2017, 5, 4384-4396. 
(97) Dohy, D.; Lucazeau, G.; Revcolevschi, A. Raman Spectra and Valence Force Field of Single-Crystalline $\beta$ Ga2O3. J. Solid State Chem. 1982, 45, 180-192.

(98) Zhou, J.; Chen, L.; Wang, Y.; He, Y.; Pan, X.; Xie, E. An Overview on Emerging Photoelectrochemical Self-Powered Ultraviolet Photodetectors. Nanoscale 2016, 8, 50-73.

(99) Qiao, H.; Huang, Z.; Ren, X.; Liu, S.; Zhang, Y.; Qi, X.; Zhang, H. Self-Powered Photodetectors Based on 2D Materials. Adv. Opt. Mater. 2020, 8, No. 1900765.

(100) Tian, W.; Wang, Y.; Chen, L.; Li, L. Self-Powered Nanoscale Photodetectors. Small 2017, 13, No. 1701848.

(101) Petroni, E.; Lago, E.; Bellani, S.; Boukhvalov, D. W.; Politano, A.; Gürbulak, B.; Duman, S.; Prato, M.; Gentiluomo, S.; OropesaNuñez, R.; et al. Liquid-Phase Exfoliated Indium-Selenide Flakes and Their Application in Hydrogen Evolution Reaction. Small 2018, 14, No. 1800749.

(102) Gray, J. L. The Physics of the Solar Cell. In Handbook of Photovoltaic Science and Engineering; John Wiley \& Sons, Ltd: Chichester, U.K., 2011; pp 82-129.

(103) Zhang, Q.; Jie, J.; Diao, S.; Shao, Z.; Zhang, Q.; Wang, L.; Deng, W.; Hu, W.; Xia, H.; Yuan, X.; et al. Solution-Processed Graphene Quantum Dot Deep-UV Photodetectors. ACS Nano 2015, 9, 1561-1570.

(104) Chitara, B.; Krupanidhi, S. B.; Rao, C. N. R. Solution Processed Reduced Graphene Oxide Ultraviolet Detector. Appl. Phys. Lett. 2011, 99, No. 113114.

(105) Gomathi, P. T.; Sahatiya, P.; Badhulika, S. Large-Area, Flexible Broadband Photodetector Based on ZnS-MoS 2 Hybrid on Paper Substrate. Adv. Funct. Mater. 2017, 27, No. 1701611.

(106) Sahatiya, P.; Jones, S. S.; Badhulika, S. 2D MoS2-Carbon Quantum Dot Hybrid Based Large Area, Flexible UV-Vis-NIR Photodetector on Paper Substrate. Appl. Mater. Today 2018, 10, 106114.

(107) Shelke, N. T.; Karche, B. R. Hydrothermal Synthesis of WS2/ RGO Sheet and Their Application in UV Photodetector. J. Alloys Compd. 2015, 653, 298-303.

(108) Bellani, S.; Ghadirzadeh, A.; Meda, L.; Savoini, A.; Tacca, A.; Marra, G.; Meira, R.; Morgado, J.; Di Fonzo, F.; Antognazza, M. R. Hybrid Organic/Inorganic Nanostructures for Highly Sensitive Photoelectrochemical Detection of Dissolved Oxygen in Aqueous Media. Adv. Funct. Mater. 2015, 25, 4531-4538.

(109) María Girón, R.; Marco-Martínez, J.; Bellani, S.; Insuasty, A.; Comas Rojas, H.; Tullii, G.; Antognazza, M. R.; Filippone, S.; Martín, N. Synthesis of Modified Fullerenes for Oxygen Reduction Reactions. J. Mater. Chem. A 2016, 4, No. 14284.

(110) Aguirre, J.; Medrano, N.; Calvo, B.; Celma, S. Lock-in Amplifier for Portable Sensing Systems. Electron. Lett. 2011, 47, No. 1172.

(111) Yue, Z.; Lisdat, F.; Parak, W. J.; Hickey, S. G.; Tu, L.; Sabir, N.; Dorfs, D.; Bigall, N. C. Quantum-Dot-Based Photoelectrochemical Sensors for Chemical and Biological Detection. ACS Appl. Mater. Interfaces 2013, 2800-2814.

(112) Zen, J. M.; Song, Y. S.; Chung, H. H.; Hsu, C. T.; Kumar, A. S. Photoelectrochemical Oxygen Sensor Using Copper-Plated ScreenPrinted Carbon Electrodes. Anal. Chem. 2002, 74, 6126-6130.

(113) Mauritz, K. A.; Moore, R. B. State of Understanding of Nafion. Chem. Rev. 2004, 104, 4535-4585.

(114) Holdcroft, S. Fuel Cell Catalyst Layers: A Polymer Science Perspective. Chem. Mater. 2014, 26, 381-393.

(115) Zhang, C. J.; Liang, M.; Park, S.-H.; Lin, Z.; Seral-Ascaso, A.; Wang, L.; Pakdel, A.; Coileáin, C. Ó.; Boland, J.; Ronan, O.; et al. Extra Lithium-Ion Storage Capacity Enabled by Liquid-Phase Exfoliated Indium Selenide Nanosheets Conductive Network. Energy Environ. Sci. 2020, 13, 2124-2133.

(116) Najafi, L.; Bellani, S.; Castelli, A.; Arciniegas, M. P.; Brescia, R.; Oropesa-Nuñez, R.; Martín-García, B.; Serri, M.; Drago, F.; Manna, L.; et al. Octapod-Shaped CdSe Nanocrystals Hosting Pt with High Mass Activity for the Hydrogen Evolution Reaction. Chem. Mater. 2020, 32, 2420-2429.
(117) Rojas, H. C.; Bellani, S.; Fumagalli, F.; Tullii, G.; Leonardi, S.; Mayer, M. T.; Schreier, M.; Grätzel, M.; Lanzani, G.; Di Fonzo, F.; et al. Polymer-Based Photocathodes with a Solution-Processable Cuprous Iodide Anode Layer and a Polyethyleneimine Protective Coating. Energy Environ. Sci. 2016, 9, 3710-3723.

(118) Bellani, S.; Antognazza, M. R.; Bonaccorso, F. Carbon-Based Photocathode Materials for Solar Hydrogen Production. Adv. Mater. 2019, 31, No. 1801446.

(119) Steier, L.; Bellani, S.; Rojas, H. C.; Pan, L.; Laitinen, M.; Sajavaara, T.; Di Fonzo, F.; Grätzel, M.; Antognazza, M. R.; Mayer, M. T. Stabilizing Organic Photocathodes by Low-Temperature Atomic Layer Deposition of TiO 2. Sustainable Energy Fuels 2017, 1, 1915-1920.

(120) Fumagalli, F.; Bellani, S.; Schreier, M.; Leonardi, S.; Rojas, H. C.; Ghadirzadeh, A.; Tullii, G.; Savoini, A.; Marra, G.; Meda, L.; et al. Hybrid Organic-Inorganic H 2 -Evolving Photocathodes: Understanding the Route towards High Performance Organic Photoelectrochemical Water Splitting. J. Mater. Chem. A 2016, 4, 21782187.

(121) Peng, L.; Hu, L.; Fang, X. Low-Dimensional Nanostructure Ultraviolet Photodetectors. Adv. Mater. 2013, 25, 5321-5328.

(122) Ni, P.-N.; Shan, C.-X.; Wang, S.-P.; Li, B.-H.; Zhang, Z.-Z.; Zhao, D.-X.; Liu, L.; Shen, D.-Z. Enhanced Responsivity of Highly Spectrum-Selective Ultraviolet Photodetectors. J. Phys. Chem. C 2012, 116, 1350-1353.

(123) Ates, E. S.; Kucukyildiz, S.; Unalan, H. E. Zinc Oxide Nanowire Photodetectors with Single-Walled Carbon Nanotube Thin-Film Electrodes. ACS Appl. Mater. Interfaces 2012, 4, 51425146.

(124) Inamdar, S. I.; Rajpure, K. Y. High-Performance MetalSemiconductor-Metal UV Photodetector Based on Spray Deposited ZnO Thin Films. J. Alloys Compd. 2014, 595, 55-59.

(125) Chen, H.; Liu, K.; Hu, L.; Al-Ghamdi, A. A.; Fang, X. New Concept Ultraviolet Photodetectors. Mater. Today 2015, 18, 493502.

(126) Peng, Q.; Xiong, R.; Sa, B.; Zhou, J.; Wen, C.; Wu, B.; Anpo, M.; Sun, Z. Computational Mining of Photocatalysts for Water Splitting Hydrogen Production: Two-Dimensional InSe-Family Monolayers. Catal. Sci. Technol. 2017, 7, 2744-2752.

(127) Sun, L.; Cui, Y.; Peng, L.; Du, J.; Wang, S.; Huang, Y. TwoDimensional Blue-Phosphorene-Phase Germanium Monochalcogenide Photocatalysts for Water Splitting: From Ultraviolet to Visible Absorption. J. Catal. 2019, 373, 67-74.

(128) Ji, Y.; Yang, M.; Dong, H.; Hou, T.; Wang, L.; Li, Y. TwoDimensional Germanium Monochalcogenide Photocatalyst for Water Splitting under Ultraviolet, Visible to near-Infrared Light. Nanoscale 2017, 9, 8608-8615. 